\title{
Existence, Uniqueness for Stochastic forest Evolution
}

\author{
Gu yinlu \\ Yinchan Energy College, \\ Yinchuan750021
}

\author{
Zhang qimin \\ Ningxia University, \\ Yinchuan750021
}

\author{
Song zhenzhen \\ Yinchan Energy College, \\ Yinchuan 750021
}

\begin{abstract}
This paper introduces random perturbations into the established system of stochastic forest evolution, and studies the system of stochastic forest evolution in Hilbert space, at the same time, using Kolmogorov's inequality and Burkholder-DavisGundy's inequality, analyzes the existence uniqueness of system of stochastic forest evolution.
\end{abstract}

Key words-Existence; uniqueness; stochastic forest evolution system; It o equation

\section{INTRODUCTION}

There has been much recent interest in application of deterministic age-dependent mathematical models in population dynamics. Population system are often subject to environment noise[1,2].For example, Cushing[3], Henson and Cushing[4] investigate hierarchical age-dependent populations with intra-specific competition or predation. Allen and Thrasher[5] consider vaccination strategies in age-dependent populations. In addition, Pollard[8],Block and Allen[9]study the effects of adding stochastic terms to discrete-time agedependent models that employ Leslie matrices.

Consider the following forest evolution system:

$$
\left\{\begin{array}{l}
\frac{\partial p}{\partial a}+\frac{\partial p}{\partial t}=-\mu(t, a) P+f(t, p), \operatorname{in} Q=(0, A) \times(0, T), \\
P(0, a)=P_{0}(a), \quad \operatorname{in}[0, A], \\
P(t, 0)=\gamma(t) \beta(t) \int_{0}^{A} \beta(t, a) P(t, a) d a, \quad \operatorname{in}[0, T],
\end{array}\right.
$$

where $P(t, a)$ is the age-area distribution density of forest. $\beta(t)$ is the ratio of reforested area to cut area $\gamma(t)$ is the reforestation percentage. By $\mu(t, a)$ is denoted the cut ratio.

$f(t, p)$ denotes affects external environment for system, it is a reduction of area because forest fires and denudation .

Suppose that $-\mu(t, a) P+f(t, p)$ is stochastically perturbed with

$$
-\mu(t, a) P+f(t, p) \rightarrow-\mu(t, a) P+f(t, p)+g(t, P) \dot{\omega}(t),
$$

Here $\dot{\omega}(t)$ is white noise. Then this environmentally perturbed system may be described by the It $o$ equation

$$
\left\{\begin{array}{lc}
d_{t} P=-\frac{\partial p}{\partial a} d t-\mu(t, a) P d t+f(t, p) d t+g(t, P) d \omega_{t}, & \text { in } Q=(0, A) \times(0, T) \\
P(0, a)=P_{0}(a), & \operatorname{in}[0, A] \\
P(t, 0)=\gamma(t) \beta(t) \int_{0}^{A} \beta(t, a) P(t, a) d a, & \operatorname{in}[0, T]
\end{array}\right.
$$

$d_{t} P$ is the differential of $P$ relative to $t$,i.e, $d_{t} P=(\partial P / \partial t) d t$

A new stochastic differential equation model (2) a forest evolution dynamic system. is derived. It is an extension of $\operatorname{Eq}(1)$.

In this paper, we shall discussion the existence, uniqueness for a forest evolution dynamic system Eq.(2).

\section{PRELIMINARIES}

Let

$$
\begin{aligned}
& V=H^{1}([0, A]) \\
& \equiv\left\{\varphi \mid \varphi \in L^{2}([0, A]), \frac{\partial \varphi}{\partial x_{i}} \in L^{2}([0, A]),\right. \\
&\left.\quad \text { where } \frac{\partial \varphi}{\partial x_{i}} \text { is generalized partial derivatives }\right\} .
\end{aligned}
$$

$V$ is a Sobolev space. $H=L^{2}([0, A])$ such that $V \rightarrow H \equiv H^{\prime} \rightarrow V^{\prime}$.

$V^{\prime}$ is the dual space of $V$.We denote by $\|\|,.|$.$| and \|.\|_{*}$ the norms in $V, H$ and $V^{\prime}$ respectively ;by $\langle.,$.$\rangle the duality$ product between $V, V^{\prime}$, and by $(.,$.$) the scalar product in$ $H$, and $m$ a constraint such that $m|x| \leq m\|x\| \quad \forall x \in V$.

Let $\omega_{t}$ be a Wiener process defined on complete probability space $(\Omega, F, \mathrm{P})$, and taking its values in the separable Hilbert space $\mathrm{K}$, with increment covariance operator $W$.Let $\left(F_{t}\right)_{t \geq 0}$ be the $\sigma$ - algebra generated by $\left\{\omega_{s}, 0 \leq s \leq t\right\}$, then $\omega_{t}$ is a martingale relative to $\left(F_{t}\right)_{t \geq 0}$ and we have the following representation of $\omega_{t}$ :

$\omega_{t}=\sum_{i=1}^{\infty} \beta_{i}(t) e_{i},\left\{e_{i}\right\}_{i \geq 1}$ is an orthonormal set of eigenvectors of $W$, $\beta_{i}(t)$ are mutually independent real Wiener processes with incremental covariance $\lambda_{i}>0, W e_{i}=\lambda_{i} e_{i}$ and $\operatorname{tr} W=\sum_{i=1}^{\infty} \lambda_{i}<\infty$

('tr' denotes the trace of an operator [13]). For an operator $B \in \Gamma(K, H)$ be the space of all bounded linear operators from $K$ into $H$, we denote by $\|B\|_{2}$ its Hilbert-Schmidt norm, i.e.

$$
\|B\|_{2}^{2}=\operatorname{tr}\left(B W B^{T}\right) \text {. }
$$


In this paper, $\omega_{t}$ is a real standard Wiener process. Let $C=C([0, T], H)$ be the space of all continuous function from $[0, T]$ into $H$ with sup-norm $\|\psi\|_{C}=\sup _{0 \leq s \leq T}|\psi(s)|, L_{V}^{P}=L^{P}([0, T] ; V)$ and $L_{H}^{P}=L^{P}([0, T] ; H)$.

Consider the following nonlinear stochastic equation:

$$
\left\{\begin{array}{rr}
P_{t}=P_{0}-\int_{0}^{t} \frac{\partial P_{s}}{\partial \alpha} d s-\int_{0}^{t} \mu(s, a) P_{s} d s & \\
\quad+\int_{0}^{t} f\left(s, P_{s}\right) d s+\int_{0}^{t} g\left(s, P_{s}\right) d \omega_{s}, & \forall t \in[0, T] \\
P(t, 0)=\gamma(t) \beta(t) \int_{0}^{A} \beta(t, a) P_{t} d a & \forall t \in[0, T]
\end{array}\right.
$$

Where $P_{t}=P(t, a), P_{0}=P(0, a)$.

The objective in this paper is that, we hopefully find a unique process $P_{t} \in I^{P}(0, T ; V) \cap L^{2}(\Omega ; C(0, T ; H))$, such that $\left(^{*}\right)$ hold.

For this objective, we assume the following conditions are satisfied: $\mu(t, a), \beta(t, a)$ are nonnegative measurable, and

$$
\left\{\begin{array}{l}
0 \leq \mu_{0} \leq \mu(t, a)<\infty \quad \text { in } Q \\
0 \leq \beta(t) \leq \bar{\beta}<\infty \quad \text { in } Q \\
0 \leq \gamma(t) \leq \bar{\gamma}<\infty \quad \text { in } Q .
\end{array}\right.
$$

Let $f(t, \cdot): L_{H}^{2} \rightarrow H$ be a family operator's defined a.e.t. and satisfy:

(a.1) $f(t, 0)=0$;

(a.2) $\exists k_{1}>0$ such that

$|f(t, y)-f(t, x)| \leq k_{1}\|y-x\|_{c}, \forall x, y \in C$, a.e.t.

Let $g(t):, L_{H}^{2} \rightarrow \Gamma(K, H)$, the family of nonlinear operator defined a.e.t., $g(t, x) \in \Gamma(K, H)$ and satisfy

(b.1) $g(t, 0)=0$;

(b.2) there exists $k_{2}>0$ such that

$$
\|g(t, y)-g(t, x)\|_{2} \leq k_{2}\|y-x\|_{c} \quad \forall x, y \in C, \quad \text { a.e.t. }
$$

$f(t, v)$ and $g(t, v)$ are Lebergue miserable $\forall v \in L_{H}^{2}$, satisfying following condition $(\mathrm{H})$ :

There exist constants $\alpha>0, \xi>0, \lambda \in R$, and a non-negative continuous function $\gamma(t), t \in R_{+}$, such that

$2\langle f(t, v), v\rangle+\|g(t, v)\|_{2}^{2} \leq-\alpha\|v\|^{2}+\lambda|v|^{2}+\gamma(t) e^{-\xi t}, \quad v \in V$, a.e.t.,

Where, for arbitrary $\delta>0, \gamma(t)$ satisfies $\gamma(t)=o\left(e^{\xi t}\right)$, as $t \rightarrow \infty$, i.e., $\lim _{t \rightarrow \infty} \gamma(t) / e^{\xi t}=0$

Remark:

Observe that, owing to continuity and sub exponential growth of the term, there exists a positive constant $\bar{\gamma}$ such that $\gamma(t) e^{\xi t} \leq \bar{\gamma}$ for all $t \in R^{+}$.As a consequence, $(\mathrm{H})$ implies

$$
2\langle f(t, v), v\rangle+\|g(t, v)\|_{2}^{2} \leq-\alpha\|v\|^{2}+\lambda|v|^{2}+\bar{\gamma}, \quad v \in V, \quad \text { a.e.t. }
$$

\section{EXISTENCE AND UNIQUENESS OF SOLUTIONS}

A

Now we shall prove that there exists at most one solution of $\left(^{*}\right)$.This result will be deduced mainly from It $O$ formula.

Theorem 3.1 Assume the preceding hypotheses hold. Then exists at most one solution of $\left(^{*}\right)$ in $I^{2}(0, T ; V) \cap L^{2}(\Omega ; C(0, T ; H))$ .Proof: Suppose $P_{1 t}, P_{2 t} \in I^{2}(0, T ; V) \cap L^{2}(\Omega ; C(0, T ; H))$ are two solutions of (*). Then, applying It $\hat{o}$ formula to $\left|P_{1 t}-P_{2 t}\right|^{2}$,we obtain

$$
\begin{aligned}
\left|P_{1 t}-P_{2 t}\right|^{2} & =2 \int_{0}^{t}\left\langle-\frac{\partial P_{1 s}}{\partial a}+\frac{\partial P_{2 s}}{\partial a}-\mu(s, a)\left(P_{1 s}-P_{2 s}\right), P_{1 s}-P_{2 s}\right\rangle d s \\
& +2 \int_{0}^{t}\left(f\left(s, P_{1 s}\right)-f\left(s, P_{2 s}\right), P_{1 s}-P_{2 s}\right) d s \\
& +2 \int_{0}^{t}\left(P_{1 s}-P_{2 s},\left(g\left(s, P_{1 s}\right)-g\left(s, P_{2 s}\right)\right) d \omega_{s}\right) \\
& +\int_{0}^{t}\left\|g\left(s, P_{1 s}\right)-g\left(s, P_{2 s}\right)\right\|_{2}^{2} d s
\end{aligned}
$$

Therefore, we get that

$$
\begin{aligned}
\left|P_{1 t}-P_{2 t}\right|^{2} \leq & A(\bar{\gamma} \bar{\beta} \bar{\mu})^{2} \int_{0}^{t}\left|P_{1 s}-P_{2 s}\right|^{2} d s \\
& +2 \int_{0}^{t}\left|P_{1 s}-P_{2 s} \| f\left(s, P_{1 s}\right)-f\left(s, P_{2 s}\right)\right| d s-2 \mu_{0} \int_{0}^{t}\left|P_{1 s}-P_{2 s}\right|^{2} d s \\
& +\int_{0}^{t}\left\|g\left(s, P_{1 s}\right)-g\left(s, P_{2 s}\right)\right\|_{c}^{2} d s+2 \int_{0}^{t}\left(P_{1 s}-P_{2 s},\left(g\left(s, P_{1 s}\right)-g\left(s, P_{2 s}\right)\right)\right) d \omega_{s} .
\end{aligned}
$$

Now, it follows from (a.2) and (b.2) that for any $t \in[0, t]$

$$
\begin{aligned}
& E \sup _{0 \leq s \leq t}\left|P_{1 s}-P_{2 s}\right|^{2} \\
& \leq\left(\left|A(\bar{\gamma} \bar{\beta} \bar{\mu})^{2}-2 \mu_{0}\right|+1\right) \int_{0}^{t} E\left|P_{1 s}-P_{2 s}\right|^{2} d s+\left(k_{1}^{2}+k_{2}^{2}\right) \int_{0}^{t} E\left\|P_{1 s}-P_{2 s}\right\|_{C}^{2} d s \\
& +2 E \sup _{0 \leq s \leq t} \int_{0}^{s}\left(P_{1 r}-P_{2 r},\left(g\left(r, P_{1 r}\right)-g\left(r, P_{2 r}\right)\right) d \omega_{r}\right) .
\end{aligned}
$$

However, by Burkholder-Davis-Gundy's inequality, $K>0$ we have

$$
\begin{aligned}
& E \sup _{0 \leq s \leq t}\left|P_{1 s}-P_{2 s}\right|^{2} \\
& \leq 2\left(\left|A(\bar{\gamma} \bar{\beta} \bar{\mu})^{2}-2 \mu_{0}\right|+1+k_{1}^{2}+k_{2}^{2}+2 K k_{2}^{2}\right) \\
& \times \int_{0}^{t} E \sup _{0 \leq s \leq t}\left|P_{1 r}-P_{2 r}\right|^{2} d s, \forall t \in[0, T] .
\end{aligned}
$$

Now, Gronwall's lemma obviously implies uniqueness.

\section{$B$}

\section{Existence of strong solutions}

In order to prove the existence of solution for Eq.(*), we shall first prove the following lemmas.

We consider the equations

$$
\begin{aligned}
P_{t}^{1} & =P_{0}+\int_{0}^{t}\left[-\frac{\partial P^{1}}{\partial a}-\frac{A(\bar{\gamma} \bar{\beta} \bar{\mu})^{2}}{2} P_{s}^{1}\right] d s, t \in[0, T], \\
2 E \int_{0}^{t}\left|f\left(P_{s}^{n}\right)-f\left(P_{s}^{n-1}\right)\right|\left|P_{s}^{n+1}-P_{s}^{n}\right| d s & \\
& \leq \frac{1}{4 T} E \int_{0}^{t}|| P_{s}^{n+1}-P_{s}^{n}|d s|+4 k_{1}^{2} T E \int_{0}^{t}|| P_{s}^{n}-P_{s}^{n-1} \|_{C}^{2} d s \\
& \leq \frac{1}{4} E\left[\sup _{0 \leq r s t}\left|P_{r}^{n+1}-P_{r}^{n}\right|\right]+4 k_{1}^{2} T \int_{0}^{t} E\left[\sup _{0 \leq r s s}\left|P_{r}^{n}-P_{r}^{n-1}\right|^{2}\right] d s .
\end{aligned}
$$




$$
P^{1}(t, 0)=\gamma(t) \beta(t) \int_{0}^{A} \beta(t, a) P_{t}^{1} d a, t \in[0, T],
$$

$$
\begin{aligned}
P_{t}^{n+1}= & P_{0}+\int_{0}^{t}\left[-\frac{\partial P_{s}^{n+1}}{\partial a}-\frac{A(\gamma \beta \mu)^{2}}{2} P_{s}^{n+1}\right] d s+\int_{0}^{t} \frac{A(\gamma \beta \mu)^{2}}{2} P_{s}^{n} d s-\int_{0}^{t} \mu(s, a) P_{s}^{n} d s \\
+ & \int_{0}^{t} f\left(s, P_{s}^{n}\right) d s+\int_{0}^{t} g\left(s, P_{s}^{n}\right) d \omega_{s}, t \in[0, T], \quad \forall n \geq 1, \\
& P^{n+1}(t, 0)=\gamma(t) \beta(t) \int_{0}^{A} \mu(t, a) P_{t}^{n+1} d a, t \in[0, T], \forall n \geq 1 .
\end{aligned}
$$

Lemma $3.1_{\left\{P_{t}^{n}\right\}}$ is a Cauchy sequence in $L^{2}(\Omega ; C(0, T ; H))$.

Proof: For $n>1$ and the process $P_{t}^{n+1}-P_{t}^{n}$, it following from Ito $s$ formula:

$$
\begin{aligned}
\left|P_{t}^{n+1}-P_{t}^{n}\right|^{n} & =2 \int_{0}^{t}\left(-\frac{\partial P_{s}^{n+1}}{\partial a}+\frac{\partial P_{s}^{n}}{\partial a}, P_{s}^{n+1}-P_{s}^{n}\right) d s-2 \int_{0}^{t}\left(\mu(s, a)\left(P_{s}^{n}-P_{s}^{n+1}\right), P_{s}^{n+1}-P_{s}^{n}\right) d s \\
& -A(\bar{\gamma} \bar{\beta} \bar{\mu})^{2} \int_{0}^{t} P_{s}^{n+1}-\left.P_{s}^{n}\right|^{2} d s+A(\bar{\gamma} \bar{\beta} \bar{\mu})^{2} \int_{0}^{t}\left(P_{s}^{n+1}-P_{s}^{n}, P_{s}^{n}-P_{s}^{n-1}\right) d s
\end{aligned}
$$

$+2 \int_{0}^{t}\left(f\left(P_{s}^{n}\right)-f\left(P_{s}^{n-1}\right), P_{s}^{n+1}-P_{s}^{n}\right) d s+2 \int_{0}^{t}\left(P_{s}^{n+1}-P_{s}^{n},\left(g\left(P_{s}^{n}\right)-g\left(P_{s}^{n-1}\right)\right) d \omega_{s}\right)+\int_{0}^{t}\left\|g\left(P_{s}^{n}\right)-g\left(P_{s}^{n-1}\right)\right\|_{2}^{2} d s$, Where, by definition, $P_{t}^{n}:=P^{n}(t, a), f\left(P_{t}^{n}\right):=f\left(t, P_{t}^{n}\right)$

and $g\left(P_{t}^{n}\right):=g\left(t, P_{t}^{n}\right)$

$\left|P_{t}^{n+1}-P_{t}^{n}\right|^{2}$

$\leq\left|A(\bar{\gamma} \bar{\beta} \bar{\mu})^{2}-2 \mu_{0}\right| \int_{0}^{t}\left|P_{s}^{n+1}-P_{s}^{n}\right|\left|n_{s}-P_{s}^{n-1}\right| d s+2 \mid \int_{0}^{t}\left(P_{s}^{n+1}-P^{n},\left(g\left(P_{s}^{n}\right)-g\left(P_{s}^{n-1}\right)\right) d \omega_{s} \mid\right.$

$+2 \int_{0}^{t}\left|f\left(P_{s}^{n}\right)-f\left(P_{s}^{n-1}\right)\right|\left|P_{s}^{n+1}-P_{s}^{n}\right| d s+\int_{0}^{t}\left\|g\left(P_{s}^{n}\right)-g\left(P_{s}^{n-1}\right)\right\|_{2}^{2} d s$

It is easy to deduce.Consequently, (9) yields

$$
\begin{aligned}
& E\left[\sup _{0 \leq \theta \leq t}\left|P_{\theta}^{n+1}-P_{\theta}^{n}\right|^{2}\right] \\
& \leq\left|A(\bar{\gamma} \bar{\mu} \bar{\mu})^{2}-2 \mu_{0}\right| E \int_{0}^{t}\left|P_{s}^{n+1}-P_{s}^{n} \| P_{s}^{n}-P_{s}^{n-1}\right| d s \\
& +2 E\left[\sup _{0 \leq \theta \leq t}\left|\int_{0}^{\theta}\left(P_{s}^{n+1}-P_{s}^{n},\left(g\left(P_{s}^{n}\right)-g\left(P_{s}^{n-1}\right)\right) d \omega_{s}\right)\right|\right.
\end{aligned}
$$$$
+2 E \int_{0}^{t}\left|f\left(P_{s}^{n}\right)-f\left(P_{s}^{n-1}\right)\left\|P_{s}^{n+1}-P_{s}^{n} \mid d s+E \int_{0}^{t}\right\| g\left(P_{s}^{n}\right)-g\left(P_{s}^{n-1}\right) \|_{2}^{2} d s\right] .
$$

On the other hand, we can get from (b.2)

$$
E \int_{0}^{t}\left\|g\left(P_{s}^{n}\right)-g\left(P_{s}^{n-1}\right)\right\|_{2}^{2} d s \leq k_{2}^{2} E \int_{0}^{t} \sup _{0 \leq r \leq s}\left|P_{r}^{n}-P_{r}^{n-1}\right|^{2} d s .
$$

In a similar manner, from (a.2) we can obtain

$$
\begin{aligned}
& 2 E \int_{0}^{t}\left|f\left(P_{s}^{n}\right)-f\left(P_{s}^{n-1}\right)\right|\left|P_{s}^{n+1}-P_{s}^{n}\right| d s \\
& \quad \leq \frac{1}{4 T} E \int_{0}^{t}|| P_{s}^{n+1}-P_{s}^{n}|d s|+4 k_{1}^{2} T E \int_{0}^{t}|| P_{s}^{n}-P_{s}^{n-1} \|_{C}^{2} d s \\
& \quad \leq \frac{1}{4} E\left[\sup _{0 \leq r \leq t}\left|P_{r}^{n+1}-P_{r}^{n}\right|\right]+4 k_{1}^{2} T \int_{0}^{t} E\left[\sup _{0 \leq r \leq s}\left|P_{r}^{n}-P_{r}^{n-1}\right|^{2}\right] d s .
\end{aligned}
$$

Now, Burkholder-Davis-Gundy's inequality implies

If we set

$$
\begin{aligned}
2 E & {\left[\sup _{0 \leq r \leq t}\left|\int_{0}^{r}\left(P_{s}^{n+1}-P_{s}^{n},\left(g\left(P_{s}^{n}\right)-g\left(P_{s}^{n-1}\right)\right)\right) d \omega_{s}\right|\right] } \\
& \leq 6 E\left[\left(\sup _{0 \leq r \leq t}\left|P_{r}^{n+1}-P_{r}^{n}\right|^{2}\right) \int_{0}^{t}\left|g\left(P_{s}^{n}\right)-g\left(P_{s}^{n-1}\right)\right|_{2}^{2}\right]^{1 / 2} \\
& \leq \frac{1}{4} E\left[\sup _{0 \leq r \leq t}\left|P_{r}^{n+1}-P_{r}^{n}\right|^{2}\right]+72 k_{2}^{2} \int_{0}^{t} E\left[\sup _{0 \leq r \leq s}\left|P_{r}^{n}-P_{r}^{n-1}\right|^{2}\right] d s .
\end{aligned}
$$

$$
\varphi^{n}(t)=E\left[\sup _{0 \leq \theta \leq t}\left|P_{\theta}^{n+1}-P_{\theta}^{n}\right|^{2}\right]
$$

Then from (10)-(13), it could be deduced that there exists a positive constant $c>0$ such that

$$
\varphi^{n}(t) \leq \frac{3}{4} \varphi^{n}(t)+c \int_{0}^{t} \varphi^{n-1}(s) d s
$$

consequently there exists $k>0$ such that

$$
\varphi^{n}(t) \leq k \int_{0}^{t} \varphi^{n-1}(s) d s .
$$

By iteration from (16), we get

$$
\varphi^{n}(t) \leq \frac{K^{n-1} T^{n-1}}{(n-1) !} \varphi^{1}(T) \quad \forall n>1 \quad \forall t \in[0, T] .
$$

Therefore

$E\left[\sup _{0 \leq \theta \leq T}\left|P_{\theta}^{n+1}-P_{\theta}^{n}\right|^{2}\right] \leq \frac{K^{n-1} T^{n-1}}{(n-1) !} \varphi^{1}(T) \quad \forall n>1$.

Obviously, (18) implies that $\left\{P_{t}^{n}\right\}$ is a Cauchy sequence $L^{2}(\Omega ; C(0, T ; H))$.

Lemma 3.2 The sequence $\left\{P_{t}^{n}\right\}$ is bounded in $I^{2}(0, T ; V)$.

Proof: Indeed, applying Ito $s$ formula to $\left|P_{t}^{n}\right|^{2}$ with $n \geq 2$ immediately yields

$E\left|P^{n}(T)\right|^{2}=2 E \int_{0}^{T}<-\frac{\partial P_{s}^{n}}{\partial a}, P_{s}^{n}>d s-2 \int_{0}^{T}\left(\mu(s, a) P_{s}^{n-1}, P_{s}^{n}\right) d s$

$$
\begin{aligned}
& -A(\bar{\gamma} \bar{\beta} \bar{\mu})^{2} E \int_{0}^{T}\left|P_{s}^{n}\right|^{2} d s+E\left|P_{0}\right|^{2}+2 E \int_{0}^{T}\left(f\left(P_{s}^{n-1}\right), P_{s}^{n}\right) d s \\
& +A(\bar{\gamma} \bar{\beta} \bar{\mu})^{2} E \int_{0}^{T}\left(P_{s}^{n}, P_{s}^{n-1}\right) d s-2 E \int_{0}^{T}\left(f\left(P_{s}^{n-1}\right), P_{s}^{n-1}\right) d s \\
& +2 E \int_{0}^{T}\left(f\left(P_{s}^{n-1}\right), P_{s}^{n-1}\right) d s+E \int_{0}^{T}\left\|g\left(P_{s}^{n-1}\right)\right\|_{2}^{2} d s .
\end{aligned}
$$

Since $\left\{P^{n}\right\}$ is convergent in $L^{2}(\Omega ; C(0, T ; H))$, it will be bounded in this space. Now, it is not difficult to check that there exists positive constant $k^{\prime}>0$.We will estimate one of those terms. First, we observe that

$$
\begin{aligned}
2 E & \int_{0}^{T}\left|f\left(P_{s}^{n-1}\right)\right|\left(\left|P_{s}^{n}\right|+\left|P_{s}^{n-1}\right|\right) d s \\
& \leq 2 k_{1} E \int_{0}^{T}\left\|P_{s}^{n-1}\right\|_{C}\left(\left|P_{s}^{n}\right|+\left|P_{s}^{n-1}\right|\right) d s \\
& \leq k_{1} E \int_{0}^{T}\left[\left\|P_{s}^{n-1}\right\|_{C}^{2}+\left(\left|P_{s}^{n}\right|+\left|P_{s}^{n-1}\right|\right)^{2}\right] d s \\
& \leq T k_{1} E\left(\sup _{0 \leq \theta \leq T}\left|P_{\theta}^{n-1}\right|^{2}\right)+2 k_{1} T\left[E\left(\sup _{0 \leq \theta \leq T}\left|P_{\theta}^{n}\right|^{2}\right)+E\left(\sup _{0 \leq \theta \leq T}\left|P_{\theta}^{n-1}\right|^{2}\right)\right] \\
& =T k_{1}\left\|P_{t}^{n-1}\right\|_{L^{2}(\Omega ; C(0, T ; H))}+2 k_{1} T\left[\left\|P_{t}^{n}\right\|_{L^{2}(\Omega ; C(0, T ; H))}+\left\|P_{t}^{n-1}\right\|_{L^{2}(\Omega ; C(0, T ; H))}\right],
\end{aligned}
$$

Which, in addition to $(\mathrm{H})$, lead to the following inequality

$$
\begin{aligned}
\alpha \int_{0}^{T} E\left\|P_{s}^{n-1}\right\|^{2} d s \leq & -2 E \int_{0}^{T}\left(f\left(P_{s}^{n-1}\right), P_{s}^{n-1}\right) d s-E \int_{0}^{T}\left\|g\left(P_{s}^{n-1}\right)\right\|_{2}^{2} d s \\
& +|\lambda| T\left\|P_{t}^{n-1}\right\|_{L^{2}(\Omega ; C(0, T ; H))}^{2}+\int_{0}^{T} \gamma(s) e^{-\xi s} d s .
\end{aligned}
$$

Since $\left\{P^{n}\right\}$ is convergent in $L^{2}(\Omega ; C(0, T ; H))$. Therefore, there exist a constant $k^{\prime}$ such that $\int_{0}^{T} E\left\|P_{s}^{n-1}\right\|^{2} d s \leq k^{\prime}$

Lemma 3.2 is proved.

Theorem 3.2 Assume the preceding hypotheses and $A(\bar{\gamma} \bar{\beta} \bar{\mu})^{2}=0$ hold. Then, there exist a unique process $P_{t} \in I^{2}(0, T ; V) \cap L^{2}(\Omega ; C(0, T ; H))$ such that

$$
P_{t}=P_{0}+\int_{0}^{t}\left[\frac{\partial P_{s}}{\partial a}+f_{1}(s)\right] d s+M(t), P \quad \text { a.s. } \quad \forall t \in[0, T],
$$

Where $f_{1} \in I^{2}(0, T ; V), P_{0} \in L^{2}\left(\Omega, F_{0}, P ; H\right)$ and $M_{t}$ is an $\mathrm{H}$ valued continuous, square integriable $F_{t}$-martingale. In addition, the following energy equality also holds: $\left|P_{t}\right|^{2}=\left|P_{0}\right|^{2}+2 \int_{0}^{t}\left|\frac{\partial P_{s}}{\partial a}, P_{s}\right| d s+2 \int_{0}^{t}\left(f_{1}(s), P_{s}\right) d s+2 \int_{0}^{t}\left(P_{s}, d M_{s}\right)+\operatorname{tr}|\langle M\rangle\rangle_{t}, \quad P$ as. $\forall t \in[0, T]$, $\langle\langle M\rangle\rangle_{t}$ denotes the quadratic variation of $M_{t}$.

Proof: See Metiver and Pellaumail [14]. 
Now we are in a position to prove the existence of solution to the problem (*).

Theorem 3.3 Assume (a.1)-(a.2), (b.1)-(b.2), and (H) hold, $P_{0} \in I^{2}(0, T ; V) \cap L^{2}(\Omega C(0, T ; H))$, there exists a unique solution of the problem (*) in $g\left(P_{t}^{n}\right) \rightarrow g\left(P_{t}\right)\left(\right.$ in $\left.L^{2}\left(\Omega ; L^{\infty}(0, T ; \Gamma(K, H))\right)\right)$.

Proof: Uniqueness hold from Theorem 3.1.

By virtue of (a.1), the family $A_{1}(t, \mathrm{~g}): V \rightarrow V^{\prime}$ defined as $A_{1}\left(t, P_{t}\right)=-\frac{\partial P_{t}}{\partial a}-\frac{A(\bar{\gamma} \bar{\beta} \bar{\mu})^{2}}{2} P_{t}$, satisfies the assumptions in

Theorem 3.2. Consequently, (5)-(7) has a unique solution $P_{t}^{1} \in I^{2}(0, T ; V) \cap L^{2}(\Omega ; C(0, T ; H))$.

We note that from (a.2) and (b.2), it follows:

(i) The mapping $(t, \omega) \in(0, T) \times \Omega$ a $\quad f\left(t, P_{t}^{1}\right) \in H$ belongs to $I^{2}(0, T ; H)$;

(ii) The mapping $(t, \omega) \in(0, T) \times \Omega$ a $g\left(t, P_{t}^{1}\right) \in \Gamma(K, H)$ belongs to the space $I^{2}(0, T ; \Gamma(K, H))$ and therefore $\int_{0}^{T} g\left(t, P_{s}^{1}\right) d \omega_{s}$ is a continuous and square integrals

$F_{1}$ - martingale .

Consequently, we can use Theorem 3.2 and get that there exist a unique process

$P_{t}^{1} \in I^{2}(0, T ; V) \cap L^{2}(\Omega ; C(0, T ; H))$, which is the solution of (5)-(7) for $\mathrm{n}=1$. By recurrence, we obtain a sequence of solutions for (5)-(7), $\left\{P_{t}^{n}\right\}_{n \geq 1} \subset I^{2}(0, T ; V) \cap L^{2}(\Omega ; C(0, T ; H))$.

Now we want to prove that the sequence $\left\{P_{t}^{n}\right\}$ is convergent to a process $P_{t}$ in $I^{2}(0, T ; V) \cap L^{2}(\Omega ; C(0, T ; H))$.

which will be the solution of $(*)$.

First, we observe that Lemma 3.1implies that there exists in $P_{t} \in L^{2}(\Omega ; C(0, T ; H))$ such that $P_{t}^{n} \rightarrow P_{t}$ in $L^{2}(\Omega C(0, T ; H))$. Since

(a.2) and (b.2), Have $f\left(P_{t}^{n}\right) \rightarrow f\left(P_{t}\right)\left(i n L^{2}\left(\Omega L^{\infty}(0, T ; H)\right)\right.$ ),

and $g\left(P_{t}^{n}\right) \rightarrow g\left(P_{t}\right)\left(\right.$ in $\left.L^{2}\left(\Omega ; L^{\infty}(0, T ; \Gamma(K, H))\right)\right)$.

$$
\begin{aligned}
\text { Let } & D P_{t}=\frac{\partial P_{t}}{\partial t}+\frac{\partial P_{t}}{\partial a} . \\
\text { So } D P_{t}^{n}= & -\frac{A(\bar{\gamma} \bar{\beta} \bar{\mu})^{2}}{2} P_{t}^{n} d t-\mu(t, a) P_{t}^{n-1} d t+\frac{A(\bar{\gamma} \bar{\beta} \bar{\mu})^{2}}{2} P_{t}^{n-1} d t \\
& +f\left(t, P_{t}^{n-1}\right) d t+g\left(t, P_{t}^{n-1}\right) d \omega_{t} .
\end{aligned}
$$

By preceding analysis, we easily obtain that

$$
\left\|D P_{t}^{n}\right\|_{v^{\prime}} \leq M \leq \infty \text {. }
$$

On the other hand, by virtue of Lemma $3.2\left\{P_{t}^{n}\right\}$ has a subsequence which is weakly convergent in $I^{2}(0, T ; V)$. But, since $P_{t}^{n} \rightarrow P_{t}$ in $L^{2}(\Omega ; C(0, T ; H))$, we can assure that $P_{t}^{n} \rightarrow P_{t}$ weakly in $I^{2}(0, T ; V)$ (in the sequel, we will denote $P_{t}^{n} \rightarrow P_{t}$ in $\left.I^{2}(0, T ; V)\right)$.In conclusion, we have proved

$$
P^{n} \rightarrow P \text { in } L^{2}(\Omega ; C(0, T ; H)),
$$

$f\left(P_{t}^{n}\right) \rightarrow f\left(P_{t}\right)$ in $L^{2}\left(\Omega ; L^{\infty}(0, T ; H)\right)$,

$$
g\left(P_{t}^{n}\right) \rightarrow g\left(P_{t}\right) \text { in } L^{2}\left(\Omega ; L^{\infty}(0, T ; \Gamma(K, H))\right),
$$

$P_{t}^{n} \rightarrow P_{t} \quad$ in $I^{2}(0, T ; V)$,

\section{$D P_{t}^{n} \rightarrow h$ in $L^{2}\left(\Omega \times(0, T) ; V^{\prime}\right)$}

Since the differential operator is continuous, so $D P=h$.

Theorem 3.3 is completed.

\section{REFERENCES}

[1] Y.Kifer, Principal eigenvalues, topological pressure, and stochastic stability of equilibrium states, Israelian Journal of Mathematics 70(1990)1-47.

[2] K.Ramanan, O.Zeitouni, The quasi-stationary distribution for small randon perturbations of certain one-dimensional maps, Stochastic Processing Applications86 (1999)25-51.

[3] J.M.Cushing, The dynamics of hierarchical age-structures populations, Journal of Mathematical Biology 32(1994)705-729.

[4] S.M. Henson, J.M. Cushing, Hierarchical models of intra-specific competition: scramble versus contest, Journal of Mathematical Biology 34(1996) 755-772.

[5] L.J.S. Allen, D.B. Thrasher, The effects of vaccination in an agedependent model for vermicelli and herpes zoster,IEEE Transations ,on Automatic Control 43(1998) 779-789.

[6] J.M. Cushin, An Introduction to Structured Population Dynamics, SIAM, Philadelphia, PA, 1998.

[7] G.F.Webb, Theory of Nonlinear Age-dependent Population Dynamics, Marcell Dekker, New York, 1985.

[8] J.H. Pollard, On the use of the direct matrix product in analyzing certain stochastic population model, Biometrika 53(1966) 397-415.

[9] G.L. Block, L.J.S. Allen, Population extinction and quasi-stationary behavior in stochastic density -dependent structured models, Bulletin of Mathematical Biology 62(2000)199-228.

[10] S. Anita, Analysis and control of age-dependent population dynamics, Kluwer Academic p-ublishers, Netherlands, 2000.

[11] T. Caraballo, Existence and uniqueness of solution for non-linear stochastic partial differenti-al equations, Collection of Mathematics 42(1)(1991)51-74.

[12] X.R. Mao, Exponential Stability of Stochastic Differential Equations, Marcel Dekker, New York, 1994.

[13] E. Pardoux, Equations aux Dérive es Partiells Stochastiques Nonlineaires Monotones, Thesis, Universit e Paris Sud,1975.

[14] M. Metiver, J. Pellaumail, Stochastic Integration, Academic Press, New York, 1980. 